VII Congresso Brasileiro de Informática na Educação (CBIE 2018)

Anais do XXIX Simpósio Brasileiro de Informática na Educação (SBIE 2018)

\title{
Programando para criar objetos de aprendizagem digitais de "Ondulatória"
}

\author{
Fabrizio Honda Franzoia ${ }^{1,2}$, Fernanda Gabriela de Souza Pires ${ }^{1,2}$, Karolayne \\ Batista Teixeira $^{1,2}$, Polianny Almeida Lima ${ }^{1}$ \\ ${ }^{1}$ Licenciatura em Computação - Universidade do Estado do Amazonas (UEA) \\ Av. Darcy Vargas, 1.200- 69050-020 - Manaus - AM - Brazil \\ ${ }^{2}$ Laboratório de Tecnologias Educacionais - Universidade do Estado do Amazonas \\ Manaus, AM - Brazil \\ ${ }^{3}$ Instituto de Computação - Universidade Federal do Amazonas (UFAM) - Manaus, AM \\ - Brazil \\ \{fhf.licl7, fpires, kbt.lic\}@uea.edu.br, poliannyalmeida@gmail.com
}

Abstract. The following article presents about the development of the computational thinking in the construction of Digital Learning Objects, with methodological support in the project-based learning (PBL) in the enhancement of the learning of the theme "wave phenomenon" using the tool "Scratch" with students of the second year of high school. In the development process, the students involved built their objects with success, and the statistical analysis pointed towards positive results with the rise of performance and the fall of the post-exam variance.

Resumo. O presente artigo trata sobre o desenvolvimento do pensamento computacional na construção de Objetos Digitais de Aprendizagem (ODA), tendo como suporte metodológico a aprendizagem baseada em projeto (PBL), na potencialização da aprendizagem do tema fenômenos ondulatórios, utilizando a linguagem de programação Scratch, com alunos do $2^{\circ}$ ano do Ensino Médio. No processo de desenvolvimento, os estudantes envolvidos construíram seus objetos com sucesso e as análises estatísticas apontaram resultados positivos com o aumento dos índices de desempenho e diminuição de variância no pós-teste.

\section{Introdução}

A evolução da Ciência e da tecnologia trouxe consigo uma série de mudanças, entre elas, uma exigência cada vez maior de um protagonismo nas ações, dada a disponibilidade de ferramentas capazes de resolver problemas do dia a dia. Nesse âmbito, a rubrica que trata do Pensamento Computacional como habilidade essencial para a vida no século XXI não pode ser dispensada, muito pelo contrário, é fator condicionante, sobretudo na educação, a base da formação de todos os indivíduos ( $\mathrm{J}$. Wing, 2017; Wing \& Stanzione, 2016).

A edição do Enem 2017, na seção de Ciências da Natureza e suas tecnologias, que envolve o conteúdo de Física, contou com uma alta incidência de questões de ondulatória $(\mathrm{G} 1,2017)$. Os estudantes demonstram certa dificuldade em compreender determinados fenômenos (Silvério, 2013), o que poderia ser atenuado com a utilização 
VII Congresso Brasileiro de Informática na Educação (CBIE 2018)

Anais do XXIX Simpósio Brasileiro de Informática na Educação (SBIE 2018)

de laboratórios físicos, que tem um alto custo, ou ainda laboratórios virtuais, estes ainda podem prover simulações, ponto positivo a seu favor (De Jong, Linn, \& Zacharia, 2013). A aprendizagem do assunto Ondulatória é de fundamental importância para estudantes do $2^{\circ}$ ano do Ensino Médio, exigindo um certo grau de abstração - um dos pilares do Pensamento Computacional. Para além de assistir, o "construir" ganha uma dimensão de maior importância nos processos de aprendizagem, é o que defende o Construcionismo de Seymour Papert (2008a, 2008b, 2008c), que vem ganhando muitas comprovações empíricas ao longo do tempo (Li, Cheng, \& Liu, 2013).

O Pensamento Computacional tem papel fundamental neste processo, sendo provedor das bases iniciais para que todos sejam produtores de tecnologias digitais e não somente consumidores. Além disso, no que se refere ao desenvolvimento da aprendizagem, estimula o raciocínio lógico e oferece subsídios para o pensamento crítico (R. S. França \& P. Tedesco, 2015) . Assim, observa-se o protagonismo de quem aprende tanto no processo de construção na linguagem de programação Scratch, quanto na aprendizagem do tema específico.

Durante o desenvolvimento de objetos digitais de aprendizagem, a construção da compreensão ocorre em simultâneo com o produto (solução), havendo assim, sempre um aprimoramento do conhecimento (Maltempi, 2005). Desse modo, a proposta da pesquisa é influenciar no desenvolvimento do Pensamento Computacional, durante a criação de Objetos Digitais de Aprendizagem em Física-Ondulatória, utilizando a linguagem de programação Scratch, ao longo do trabalho se pretende avaliar a hipótese de que a produção de objetos digitais de aprendizagem utilizando o Scratch, pode gerar aprendizagem significativa.

A estrutura deste artigo, além da introdução, contempla: na seção 2, a apresentação dos trabalhos relacionados ao artigo; na seção 3, uma breve apresentação dos conceitos trabalhados e a ferramenta utilizada; na seção 4, a base teórica utilizada no desenvolvimento; na seção 5, a metodologia utilizada nas aplicações; na seção 6 são apresentados os principais resultados e suas respectivas análises; e na seção 7, a conclusão geral.

\section{Trabalhos Relacionados}

A exploração do pensamento computacional no ensino médio, através de jogos digitais, realizado por França e Tedesco (R. França \& P. Tedesco, 2015; 2015), em Pernambuco, expôs um estudo que analisou o impacto da avaliação por pares na aprendizagem do pensamento computacional, através da criação de jogos e investigou se havia um contraste entre essa avaliação e uma avaliação com abordagem tradicional, obtendo resultados médios no grupo de experimentação, em ambas as hipóteses, superior ao grupo de controle (R. França \& P. Tedesco, 2015) .

O trabalho apresentado por Carvalho, Chaimowicz \& Moro (2013), relata a experiência de implantação de um projeto cujo objetivo foi estimular o desenvolvimento do Pensamento Computacional e inserir conceitos de TI nos currículos escolares. O projeto, denominado REM - Reinventando o Ensino Médio - da Secretaria de Educação do Estado de Minas Gerais, obteve resultados de sucesso após sua primeira aplicação em 11 escolas no ano de 2012 e, posteriormente, em outras 122 escolas em 2013.

No Rio Grande do Sul, a pesquisa sobre Modelagem Computacional no Ensino de Física relatou sobre como modelos científicos podem ser implementados em sistemas 
VII Congresso Brasileiro de Informática na Educação (CBIE 2018)

Anais do XXIX Simpósio Brasileiro de Informática na Educação (SBIE 2018)

computacionais. Focando em abrir novas possibilidades na aprendizagem de Física, foi aplicada a modelagem esquemática de Halloun, trabalhada em cinco estágios: seleção, construção, validação, análise e expansão. Concluiu-se que o uso de computadores pode constituir outros obstáculos epistemológicos, se não for aplicado corretamente, pois todas as simulações no computador estão baseadas em modelagens do mundo real, levando o estudante a avaliar os conceitos envolvidos e suas relações, ressaltando a importância da visualização de fenômenos físicos (Veit \& Araujo, 2005).

\section{Pensamento Computacional um processo cognitivo}

Por anos se tem investigado a origem do conhecimento, como os homens pensam (Howard Gardner, 1995; H. Gardner, 1995) e processam as informações para reconhecimento do mundo e ação sobre o mesmo. Diante de vários estudos, chegou-se à conclusão de que a memória é essencial nesse processo, é a partir dela que virão os reconhecimentos, processamentos e consolidação de aprendizagem (R.J. Sternberg, 2016; Robert J Sternberg \& Sternberg, 2016). Dessa forma, quanto mais tempo um indivíduo dedicar-se a uma tarefa, mais significado ela irá ganhar pelo aumento de redes neurais criadas. Gonzalez (F. González, 1998; 1998), fundamentado pela Teoria Cognitiva, divide a cognição em dois campos, o primeiro trata da destreza cognitiva de ordem inferior, se refere aos processos ligados à codificação, armazenamento, recuperação e transformação da informação, e o segundo se refere aos processos mentais superiores (metacognitivos e autorreguladores), é o responsável pelo planejamento, ativação, monitoramento, avaliação e modificação dos processos de nível inferior.

O pensamento computacional é definido como processo envolvido na formulação de um problema, expressando sua solução de uma maneira que um computador ou humano possam realizar (J. Wing, 2017; J. M. Wing, 2017), ou seja, é uma nova abordagem da Teoria Cognitiva, ligada às rubricas da Ciência da Computação, a partir da compreensão de que quanto mais cedo se desenvolve o PC, maiores as possibilidades de lidar com a resolução de problemas de ordem complexa, que podem ou não ser resolvidos a partir das novas tecnologias digitais, ou desenvolvimento de outras. Tendo quatro pilares básicos como fundamento: Decomposição, Padrão, Abstração e Algoritmo. A Decomposição consiste em "quebrar" problemas em pedaços menores, tornando mais fácil de manipulá-los; 0 Reconhecimento de Padrões é analisar e observar, por uma sequência de repetição; a Abstração trata de remover partes de um problema, consideradas desnecessárias, para assim trabalhar numa solução para múltiplos problemas; e Algoritmo é caracterizado por instruções, passo a passo, de como fazer algo. O Pensamento Computacional possibilita a melhor organização do raciocínio lógico do estudante, permitindo-o ser protagonista das próprias ações, desenvolvendo soluções para os problemas que se apresentam (Teodoro \& Bhering, 2016) .

\subsection{Aprender a programar para simular Fenômenos Físicos em Scratch}

As tecnologias digitais provêm ferramentas que possibilitam relacionar conteúdos complexos a acontecimentos do dia a dia que exprimem conceitos de Física (Veit \& Araujo, 2005) . Esses experimentos podem ser desenvolvidos através da programação de algoritmos, oferecendo uma experiência mais acessível, por sua característica virtual, 
VII Congresso Brasileiro de Informática na Educação (CBIE 2018)

Anais do XXIX Simpósio Brasileiro de Informática na Educação (SBIE 2018)

podendo desencadear o interesse do estudante acerca dos fenômenos (Bell, Witten, \& Fellows, 2011).

Seymour Papert (1980) propõe que se o indivíduo utilizar meios computacionais para criação da sua aprendizagem, visualizará seus produtos e conseguirá estabelecer uma relação entre concreto e abstrato. É na abstração que está a maior força do pensamento computacional (J. Wing, 2017; J. M. Wing, 2017). A partir da produção de tecnologias próprias para aprendizagem, gerar conhecimento tanto na pesquisa aplicada ao processo da ação, quanto no processo construtivo dessas tecnologias (Reis, 2016) , em ambos os casos o indivíduo está exposto a resolução de problemas.

Existem várias ferramentas que permitem a criação de Objetos Digitais de Aprendizagem (ODA), que podem ser simulações, ferramentas de comunicação, e ferramentas de gestão de aprendizagem, que relacione aprendizagem com as ferramentas de Ciência da Computação (Clyde, 2004). O instrumento usado nessa trabalho é o Scratch2, uma linguagem com ambiente diferenciado de programação visual.

A linguagem de programação Scratch ${ }^{1}$ foi desenvolvida em 2003, pelo Lifelong Kindergarten Group do Massachusetts Institute Of Technology/MIT Media Lab, para um público alvo de 8-16 anos. Na construção dos scripts utilizam-se simples blocos de comandos, disponíveis para serem "arrastados" para a integração das linhas de comando. Desenvolvido com base na teoria de Seymour Papert (Resnick et al., 2009), permite a criação de animações, jogos, quiz, entre outros recursos de mídias - ideal para simulação dos fenômenos ondulatórios. O Scratch é uma linguagem visual que possibilita uma melhor abstração das estruturas dos algoritmos nesta faixa etária do público alvo (8 - 16 anos), visto que tais indivíduos possuem este processo (de abstração) em desenvolvimento e já estão alfabetizados.

\subsection{Construcionismo de Papert}

O construtivismo de Jean Piaget defende a ideia de que o conhecimento é construído ativamente pelos estudantes e a aprendizagem ocorre através deste processo de construção, realizando ligações cognitivas de conhecimentos prévios a novos conhecimentos, sendo guiado por um instrutor (Fino, 2004). O construcionismo é considerado, por Seymour Papert, uma extensão do construtivismo, assim " $O$ Construcionismo postula que o aprendizado ocorre especialmente quando o aprendiz está engajado em construir um produto de significado pessoal” (Maltempi, 2005)

O processo de aprendizagem é baseado na construção ativa do conhecimento pelos estudantes e, segundo Fino (2004) , “[...] educar consiste em proporcionar-lhes oportunidades de se ocuparem em atividades criativas, que alimentem aquele processo de construção de conhecimento". Seguindo esse raciocínio, a Teoria do Construcionismo de Seymour Papert retrata que a aprendizagem ocorre quando o discente constrói algo significativo, podendo ser por meios computacionais (Costa Nunes \& Santos, 2013).

\section{Métodos}

A pesquisa é do tipo experimental em que, a partir da definição do objeto de estudo, pretende analisar a influência de variáveis eletivas sobre ele, para tanto, é necessário um

\footnotetext{
${ }^{1}$ https://scratch.mit.edu/
} 
VII Congresso Brasileiro de Informática na Educação (CBIE 2018)

Anais do XXIX Simpósio Brasileiro de Informática na Educação (SBIE 2018)

espaço de trabalho minimamente delimitado a fim de averiguar os efeitos que a(s) variáveis podem causar ao objeto (Brocke \& Rosemann, 2013). Pretende-se testar as seguintes hipótese:

$\mathbf{H}_{0}$ : A utilização de uma sequência didática, que vise o desenvolvimento do pensamento computacional para criação de objetos digitais sobre Ondulatória, utilizando o Scratch, não gera impactos sobre a aprendizagem.

H1: A utilização de uma sequência didática, que vise o desenvolvimento do pensamento computacional para criação de objetos digitais sobre Ondulatória, utilizando o Scratch, gera impactos sobre a aprendizagem.

\subsection{Sequência de Atividades desenvolvidas}

As atividades desenvolvidas para este trabalho foram divididas em momentos, tendo como objetivo a criação de objetos digitais de aprendizagem (ODA), usando a linguagem de programação Scratch para a experimentação virtual dos fenômenos em Física-Ondulatória. Para isso, foi necessário um processo de imersão através de atividades cujo objetivo foi o desenvolvimento do Pensamento Computacional. Na Tabela 1, observam-se as etapas das aulas conceituais e atividades trabalhadas.

Tabela 1. Aplicações

\begin{tabular}{lll}
\hline Etapas & Conceitos trabalhados & Atividade \\
\hline Introdução a computação & $\begin{array}{l}\text { Computação; } \\
\text { Ciência da computação. }\end{array}$ & $\begin{array}{l}\text { Guiar um robô em tabuleiro } \\
\text { através de comando simples. }\end{array}$ \\
\hline Pensamento computacional & $\begin{array}{l}\text { Decomposição; } \\
\text { Padrão; } \\
\text { Abstração; } \\
\text { Algoritmo. }\end{array}$ & $\begin{array}{l}\text { Atividade de identificar essas } \\
\text { etapas através de desenho de } \\
\text { monstros }\end{array}$ \\
\hline \multirow{2}{*}{ Algoritmo } & $\begin{array}{l}\text { Algoritmo; } \\
\text { Codificação; } \\
\text { Depuração. }\end{array}$ & $\begin{array}{l}\text { Transformação de texto em } \\
\text { código e vice-versa. }\end{array}$ \\
\hline Estruturas de raciocínio lógico & $\begin{array}{l}\text { Condicional; } \\
\text { Loop de repetição; } \\
\text { Função. }\end{array}$ & $\begin{array}{l}\text { Construção de algoritmos. } \\
\text { Introdução ao uso da linguagem } \\
\text { de programação Scratch }\end{array}$ \\
\hline
\end{tabular}

Objetivando melhor aplicação do pré-teste e pós-teste, a professora da disciplina ficou responsável pelo desenvolvimento dos testes e pela atribuição de notas.

O primeiro momento consistiu em uma observação da turma, apontando a unidade amostral, composta por 42 discentes $\left(\mathrm{N}_{\mathrm{T}}=42\right)$ do $2^{\circ}$ ano do Ensino Médio, sendo $\mathrm{N}_{\mathrm{f}}=26$ (feminino) e $\mathrm{N}_{\mathrm{m}}=16$ (masculino) e uma unidade amostral de controle, composta por 26 discentes $\left(\mathrm{N}_{\mathrm{C}}=26\right)$, com faixa etária entre 15 a 17 anos. Aplicou-se um pré-teste com a finalidade de identificar quantitativamente fatores que poderiam interferir na aprendizagem dos estudantes.

No segundo momento foi executada uma remodelagem do conteúdo a ser aplicado, de acordo com os obstáculos identificados durante o primeiro momento. Definiu-se que haveriam 8 aulas de 90 minutos da disciplina de Física II: 5 aulas para aplicação de conceito e reconhecimento de linguagem de programação (02 computação desplugada para introduzir conceitos básicos de lógica de programação e 03 para linguagem de programação Scratch) e 3 aulas de desenvolvimento do ODA e 
apresentação do projeto final. Nesse processo observa-se o desenvolvimento do pensamento computacional, através da resolução de problemas e a emergência do método construtivista. As aplicações conceituais seguiram as regências conforme mostrado na Figura 1.

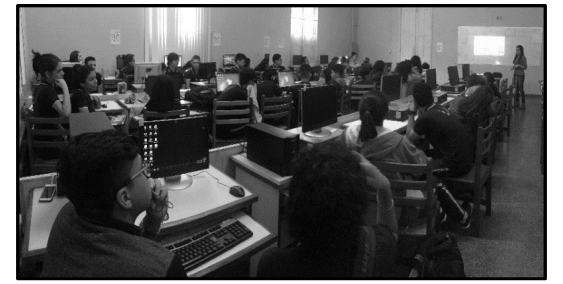

Figura 1. Aplicação da aula sobre introdução à computação.

Nesta etapa foram trabalhados os quatro pilares do pensamento computacional, em cada atividade, por meio da computação desplugada com a temática de "monstros": A primeira atividade foi a de decomposição com Monstros, os discentes tinham que decompor os rostos dos monstros; a segunda atividade envolvia o conceito de padrão, os discentes deveriam relatar os padrões encontrados nos rostos dos monstros; a terceira atividade foi acerca do conceito de abstração, na qual o discente teria que abstrair as definições que representavam as características do monstro. Através da atividade de Algoritmo, os discentes puderam descrever uma sequência de passos para compor um monstro com diferentes características e utilizar os conceitos anteriores para desenhá-lo e nomeá-lo de forma correta.

Tabela 2. Assuntos tratados em Ondulatória

\begin{tabular}{|c|c|}
\hline Ondas & Acústica \\
\hline Ondas mecânicas & Som e sua propagação \\
\hline Ondas eletromagnéticas & Intervalo acústico \\
\hline Ondas transversais & Intensidade sonora \\
\hline Ondas longitudinais & Tubos sonoros \\
\hline Reflexão & Efeito Doppler \\
\hline Refração & Reflexão do som \\
\hline Difração & \\
\hline Ressonância & \\
\hline
\end{tabular}

O terceiro momento foi caracterizado pela efetivação de 3 aulas de Introdução ao uso da linguagem de programação Scratch, para entender seu funcionamento e estruturas presentes através de atividades para melhor compreensão, fazendo uso dos computadores disponíveis no laboratório de informática.

O quarto momento foi o projeto de desenvolvimento de objetos de aprendizagem digitais, baseados nos fenômenos de ondulatória, utilizando a metodologia ativa de Aprendizagem Baseada em Projetos (PBL). Este momento ocorreu em 3 aulas: na primeira aula foram propostos os conceitos a serem utilizados que visam realizar uma pergunta motivadora que poderia ser resolvida através de pesquisas e construção de conceitos, verificando quais estudantes possuíam conhecimento acerca do assunto; 
Na segunda aula propôs-se um projeto de desenvolvimento, que consistia em relacionar o material didático exposto, seus conceitos e como poderiam ser modelados computacionalmente para criação de um ODA. Nessa etapa, pesquisas por conteúdo eram permitidas, possibilitando a realização do cumprimento do projeto.

O projeto tinha o objetivo de desenvolver Objetos Digitais de Aprendizagem, na disciplina de Física, especificamente no assunto de ondulatória. Foram formadas duplas que fizeram a escolha de trabalhar com um dos tópicos presente na Tabela 2, com o propósito de desenvolver um ODA, através da linguagem de programação Scratch, conforme ilustra a Figura 2. O Scratch foi selecionado como ferramenta por sua interface gráfica amigável e sua robustez no que diz respeito a programação, considerando que o público alvo foi de adolescentes, cuja idade permite maiores processos de abstração entretanto, por ser o primeiro contato, a ferramenta permite uma maior interatividade entre os blocos de programação e as ações a serem executadas.

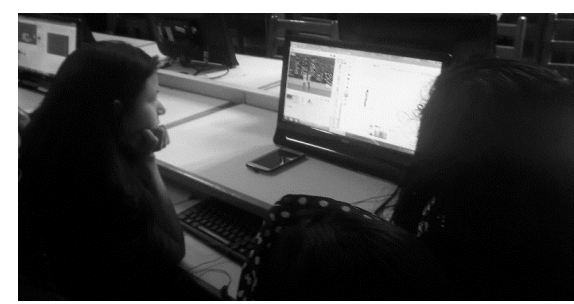

Figura 2. Desenvolvimento do Objeto de aprendizagem em Ondulatória.

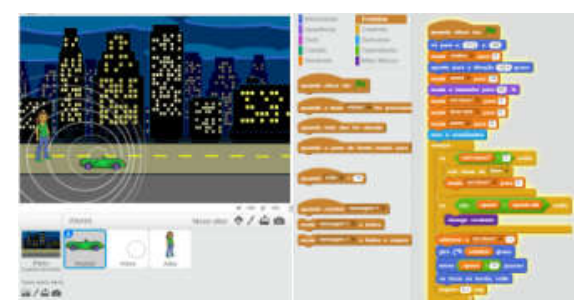

Figura 3. Objeto de aprendizagem em Ondulatória - Efeito Doppler.

A figura 3, mostra um dos ODA desenvolvidos pelos alunos acerca do fenômeno ondulatório Efeito Dopple, que consiste na simulação do som emitido por um carro em movimento com relação ao seu observador. O ODA explica o fenômeno através do uso e, durante seu desenvolvimento, foram necessários estudos do comportamento deste. $\mathrm{Na}$ terceira aula os estudantes finalizaram os seus projetos e realizaram as suas apresentações. Todos puderam ver e compartilhar os trabalhos com os colegas. As dúvidas suscitadas foram esclarecidas depois da apresentação. No quinto momento ocorreu a entrega dos Objetos Digitais de Aprendizagem dos discentes e aplicação do pós-teste para verificação da aprendizagem sobre ondulatória.

\section{Resultados e discussão dos resultados}

A média da nota do pré-teste do Grupo $\mathrm{N}_{\mathrm{T}}$ foi 7,4, sendo a nota mínima 5,3. No pósteste a nota média foi 9,0, sendo a mínima 8,1 e a máxima 9,9. Os projetos desenvolvidos e a comunicação da explicação dos processos levam a acreditar que os impactos foram positivos. A criação dos ODA, pelos alunos, são a comprovação da compreensão das estruturas lógicas de programação, considerando que os mesmos nunca tiveram contato com esse tipo de ferramenta e desconheciam os conceitos de estruturas lógicas de programação. Os testes e o diálogo sobre os Fenômenos físicos explicados podem indicar que os estudantes compreenderam o tema, pois foram capazes de encontrar uma forma lúdica de exemplificá-lo.

O desvio padrão antes da utilização da sequência didática foi 1,42 e no final de 0,51, o que demonstra uma diminuição no índice de dispersão. Conforme pode ser observado no Gráfico 1, houve uma evolução significativa nos índices da maior parte dos estudantes, entre estado inicial e estado final. 

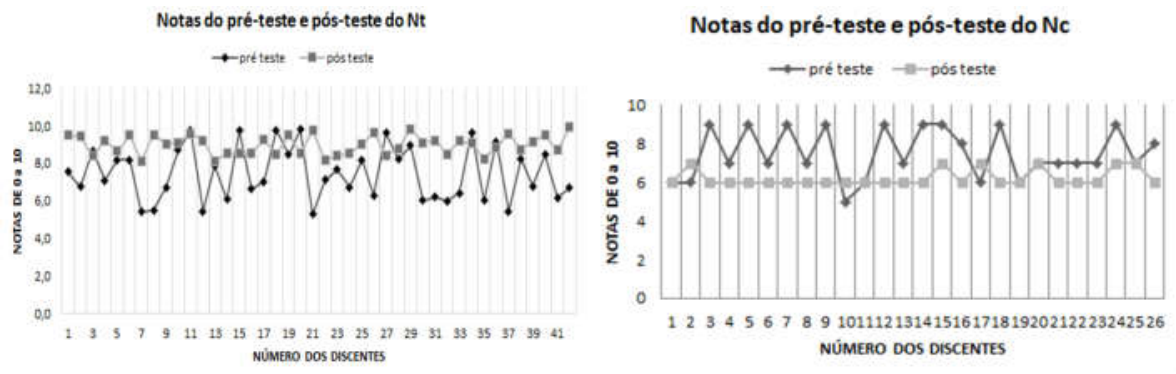

A média do grupo $\mathrm{N}_{C}$ da nota do pré-teste foi 7,5 e no pós- teste a nota média foi 6,23 , é visível que a unidade amostral $\mathrm{N}_{\mathrm{C}}$ obteve uma queda de desempenho entre préteste realizado e pós-teste, enquanto o grupo $\mathrm{N}_{\mathrm{T}}$ obteve um aumento significativo, além disso ocorreu pouca variância de desempenho no pós-teste.

Utilizou-se o teste estatístico, "T-Student”, com a finalidade de avaliar se existe diferença significativa entre o pré-teste e o pós-teste para comprovação das hipóteses nesta pesquisa. No teste de hipótese "T-Student", foram utilizadas duas amostras, presumindo variâncias diferentes para média da turma, gerando os resultados através da comparação do pré-teste e do pós-teste realizados durante a pesquisa. As aplicações dos testes obtiveram os seguintes resultados estatísticos, conforme se observa na Tabela 3.

Tabela 3. Teste T-Student: duas amostras presumindo variâncias diferentes

\begin{tabular}{lcc}
\hline & Pré-teste & Pós teste \\
\hline Média & 7,4 & 9,0 \\
Variância & 2,01 & 0,26 \\
Observações & 42 & 42 \\
$\mathrm{P}(\mathrm{T}<=\mathrm{t})$ bicaudal & & $6,50827 \mathrm{E}-15$ \\
$\mathrm{t}$ crítico bicaudal & & 2,00758377 \\
\hline
\end{tabular}

De acordo com a tabela "T-Student", por critério convencional, se o p-valor encontrado for abaixo de 0,05 a hipótese $\mathrm{H}_{0}$ é refutada comprovando $\mathrm{H}_{1}$, ao nível de $\mathrm{p}=$ 0,01. Essa diferença é considerada significante estatisticamente, validando a $\mathrm{H}_{1}$. É visível a melhora do desempenho antes e depois das aplicações, tendo como referências as notas dos testes realizados. O nível de variância da turma diminuiu, demonstrando que a mesma passou a ter uma diferença menor entre níveis de conhecimento no assunto Ondulatória. Os resultados obtidos estatisticamente confirmam a eficiência do design de aprendizagem, que visou o desenvolvimento do pensamento computacional para criação de objetos digitais de experimentações virtuais sobre Ondulatória, utilizando o Scratch.

\section{Conclusão e Trabalhos Futuros}

O pensamento computacional é uma habilidade necessária para a sociedade do século XXI, contudo, não está integrada ao currículo escolar. Nesse contexto, o foco do trabalho voltou-se para o Ensino Médio, visando disseminar o PC através da linguagem 
VII Congresso Brasileiro de Informática na Educação (CBIE 2018)

Anais do XXIX Simpósio Brasileiro de Informática na Educação (SBIE 2018)

de programação Scratch, no desenvolvimento de Objetos Digitais de Aprendizagem, na disciplina de Física abordando o tema Ondulatória.

A pesquisa obteve um resultado satisfatório com relação ao índice de aprendizagem analisado a partir da inserção de uma sequência didática que presou pelo desenvolvimento do pensamento computacional e partir disso, utilizou o Scratch para a criação de Objetos Digitais de Aprendizagem, através de uma metodologia ativa de Aprendizagem Baseada em Projetos, obtendo resultados satisfatórios. Um dos obstáculos identificados durante a pesquisa foi $\mathrm{o}$ fato de que os discentes se encontravam focados para um bom desempenho em vestibulares, para ingressarem em uma universidade pública, assim, houve uma resistência inicial quanto à utilização de novos métodos.

Para trabalhos futuros, pretende-se usar parte do Design de Aprendizagem para outros públicos, como escolas de Ensino técnico e com crianças, estimulando o desenvolvimento do pensamento computacional em faixas etárias menores.

\section{References}

Bell, T., Witten, I. H., \& Fellows, M. (2011). Computer science unplugged. ensinando ciência da computação sem o uso do computador. Tradução coordenada por Luciano Porto Barreto.

Brocke, J. V., \& Rosemann, M. (2013). Metodologia de pesquisa. Porto Alegre: AMGH Editora.

Clyde, L. A. (2004). Digital learning objects. Teacher Librarian, 31(4), 55.

Costa Nunes, M. S., \& Santos, R. P. (2013). O Construcionismo de Papert na criação de um objeto de aprendizagem e sua avaliação segundo a taxionomia de Bloom.

Carvalho, M. L. B., Chaimowicz, L., \& Moro, M. M. (2013). Pensamento computacional no ensino médio mineiro. Paper presented at the Workshop de Educação em Informática (WEI).

Jong, T., Linn, M. C., \& Zacharia, Z. C. (2013). Physical and virtual laboratories in science and engineering education. Science, 340(6130), 305-308.

Fino, C. N. (2004). Convergência entre a teoria de Vygotsky e o construtivismo/construcionismo. acessado em, 23(11), 2016.

França, R., \& Tedesco, P. (2015). Desafios e oportunidades ao ensino do pensamento computacional na educação básica no Brasil. Paper presented at the Anais dos Workshops do Congresso Brasileiro de Informática na Educação.

França, R. S., \& Tedesco, P. (2015). Explorando o pensamento computacional no ensino médio: do design à avaliação de jogos digitais. Paper presented at the Anais do XXIII Workshop sobre Educação em Computação (WEI).

G1. (2017). Enem 2017: com média de 15 questões de física por edição, prova exige ir além da decoreba.

Gardner, H. (1995). Nova ciência da mente, a-uma história da revolução cognitiva vol. 09: Edusp.

Gardner, H. (1995). Nova Ciência da Mente, A - Uma História da Revolução Cognitiva Vol. 09: EDUSP. 
VII Congresso Brasileiro de Informática na Educação (CBIE 2018)

Anais do XXIX Simpósio Brasileiro de Informática na Educação (SBIE 2018)

González, F. (1998). Metacognición y tareas intelectualmente exigentes: el caso de la resolución de problemas matemáticos. Zetetiké, 6(9), 59-88.

González, F. E. (1998). Metacognicion y tareas intelectualmente exigentes: el caso de la resolución de problemas matemáticos p. 59-87 (Segunda Parte: p. 74-87). Zetetiké, 6(1).

Li, Z. Z., Cheng, Y. B., \& Liu, C. C. (2013). A constructionism framework for designing game-like learning systems: Its effect on different learners. British Journal of Educational Technology, 44(2), 208-224.

Maltempi, M. V. (2005). Novas tecnologias e construção de conhecimento: reflexões e perspectivas. Paper presented at the Congresso Ibero-Americano De Educação Matemática.

Papert, S. (2008a). Logo: computadores e educação. São Paulo: Brasiliense, 1980. A Máquina das Crianças. Porto Alegre-RS: Artmed.

PAPERT, S. (2008b). Logo: Computadores e Educação. São Paulo: Brasiliense, 1980. _. A Máquina das Crianças. Porto Alegre-RS: Artmed.

Papert, S. (2008c). A MAQUINA DAS CRIANCAS.

Papert, S., Valente, J. A., \& Bitelman, B. (1980). Logo: computadores e educação: Brasiliense.

Reis, R. H. S., Márcia Castilho. (2016). A pesquisa-ação como estratégia de constituição de protagonismo na educação. 1 .

Resnick, M., Maloney, J., Monroy-Hernández, A., Rusk, N., Eastmond, E., Brennan, K., ... Silverman, B. (2009). Scratch: programming for all. Communications of the $A C M$, 52(11), 60-67.

Silvério, A. d. A. (2013). As dificuldades no ensino/aprendizagem da física.

Sternberg, R. J. (2016). PSICOLOGIA COGNITIVA: TRADUÇAO DA 5a EDIÇAO NORTE-AMERICANA: CENGAGE.

Sternberg, R. J., \& Sternberg, K. (2016). Cognitive psychology: Nelson Education.

Teodoro, P. V., \& Bhering, M. J. (2016). O protagonismo estudantil na elaboração de propostas pedagógicas na escola: avalicação de um estudo de caso. Ciclo Revista, 1(2).

Veit, E. A., \& Araujo, I. S. (2005). Modelagem computacional no Ensino de Física. Revista do Centro de Educação da Universidade Federal de Alagoas. CEDU-n.

Wing, J. (2017). Computational thinking's influence on research and education for all. Italian Journal of Educational Technology, 25(2), 7-14.

Wing, J. M. (2017). Computational thinking's influence on research and education for all. Italian Journal of Educational Technology, 25(2), 7-14.

Wing, J. M., \& Stanzione, D. (2016). Progress in computational thinking, and expanding the HPC community. Communications of the ACM, 59(7), 10-11. 